\title{
Non-verbal Persuasion and Communication in an Affective Agent
}

\author{
Elisabeth André, Elisabetta Bevacqua, Dirk Heylen, Radoslaw Niewiadomski, \\ Catherine Pelachaud, Christopher Peters, Isabella Poggi, and Matthias Rehm
}

\begin{abstract}
This chapter deals with the communication of persuasion. Only a small percentage of communication involves words: as the old saying goes, "it's not what you say, it's how you say it". While this likely underestimates the importance of good verbal persuasion techniques, it is accurate in underlining the critical role of non-verbal behaviour during face-to-face communication. In this chapter we restrict the discussion to body language. We also consider embodied virtual agents. As is the case with humans, there are a number of fundamental factors to be considered when constructing persuasive agents. In particular, one who wishes to persuade must appear credible, trustworthy, confident, and non-threatening. Knowing how not to behave is also a vital basis for effective persuasion. This includes resolving task constraints or other factors with the social perception considerations. These social virtual agents face many of the same problems as humans have in controlling and expressing themselves in an appropriate manner so as to establish and maintain persuasive interaction. All along the chapter, much of our discussion will handle concepts applicable both to agent and to human behaviour.
\end{abstract}

\section{Introduction}

Persuasion is a way to influence other people, that is, to make them do actions, pursue goals, that they would have not otherwise. But it differs from other ways to influence for three reasons: (1) it does not imply the use of force, but makes an appeal to the persuadee's free choice; (2) it claims that the goal/action proposed by the persuader is in the interest of the persuadee in that it is a subgoal to the persuadee's goals, and (3) it aims at influencing through communication: by letting the persuadee know that the persuader wants him to do so. Persuasion necessarily

\footnotetext{
E. André $(\bowtie)$

University of Augsburg, Augsburg, Germany

e-mail: Andre@informatik.uni-augsburg.de
} 
goes through communication: in part through argumentation, that is, words and sentences; but only a small percentage of communication involves words: as the old saying goes, "it's not what you say, it's how you say it". While this likely underestimates the importance of good verbal persuasion techniques (see the chapter by M. Guerint et al., this volume), it is accurate in underlining the critical role of nonverbal behaviour during face-to-face communication: For no matter how correct the argument or message, it is unlikely to be listened to unless the non-verbal language is congruent, communicating credibility, confidence, and trustworthiness. For example, in experiments concerning the expression of attitudes and feelings, Mehrabian (1971) found that when there was incongruence in verbal and non-verbal messages being communicated, the relative importance of messages was $7 \%$ based on verbal liking, $38 \%$ on vocal liking, and $55 \%$ on facial liking: receivers tended to predominantly favour the non-verbal aspects, in contrast to the literal meaning of the words, during the communication of attitudes and feelings.

While the environment, haptics, state of the persuadee, and appearance of persuader are also of importance, here we restrict the discussion to body language. Body language is certainly not a new topic in the domain of scientific study (Darwin, 1872), although its use in practical persuasive situations has increasingly been the focus of studies in marketing, political campaigning, and courtroom scenarios (Bernstein et al., 1994) and is becoming popularised through the publication of numerous easily accessible books (Pease and Pease, 2006). A relatively new and novel domain for the evaluation and application of body language research is that of embodied virtual agents. If embodied agents resemble humans in appearance, then they can take advantage of these extra modalities to enrich and smooth the humancomputer interaction process. These social virtual agents therefore face many of the same problems as humans have in controlling and expressing themselves in an appropriate manner so as to establish and maintain persuasive interaction - as such, much of our discussion will handle concepts applicable both to agent and to human behaviour.

As is the case with humans, there are a number of fundamental factors to be considered when constructing persuasive agents. In particular, one who wishes to persuade must appear credible, trustworthy, confident, and non-threatening. Knowing how not to behave is also a vital basis for effective persuasion. This includes resolving task constraints or other factors with the social perception considerations. For example, not looking at people due to consulting one's notes may give the impression of dishonesty; slouching due to tiredness could be misinterpreted as a sign of a lack of interest. Good behaviour does not apply solely to the speaker of course. For example, the background non-verbal behaviours made when one does not hold the floor can also have a large effect on credibility ratings (Seiter et al., 2006).

In the next section of this chapter we report on several studies on the role of nonverbal behaviours in persuasion. While Sect. 3 presents studies from the standpoint of the speaker, Sect. 4 examines the effects of persuasive non-verbal behaviours on listener's perception of speaker. Sect. 5 continues to look at the listener. It addresses the issues of backchannel signals emitted by the listener as a way of assessing the 
effect of persuasion on the listener. In the last section of this chapter we present works on virtual agents endowed with social capabilities, in particular the capacities of controlling and managing their facial behaviours and gestures.

\section{Persuasion and Emotion}

To persuade the other to do something we must convince him that this is the right thing to do; and we can do so, as Aristotle put it, through logos (rational arguments), ethos (the orator's character), and pathos (the audience's emotions): in other words, to persuade we must (1) provide good reasons to do what we propose, (2) win the interlocutor's trust, and (3) induce or evoke emotions. The first aspect, logos, is the object of the science of argumentation, which studies the possible arguments and counter-arguments that can be made explicit through verbal language; the second refers to the need for the persuader to show an image of a person who is both (a) competent in the topic dealt with (competence), in such a way that his arguments are taken at face value, and (b) one who really wants to make the interests of the persuadee (benevolence), so that the persuadee does not fear to be deceived and to get harm from doing what the persuader proposes. In this work we do not go deep into the issue of argumentation, nor in the aspect of the persuader's ethos. Here we focus on the role of emotions in persuasion. Why and how can emotions be an instrument of persuasion? Emotions are linked to goals at least in three ways:

1. Emotions monitor goals: The function of emotions is to monitor humans' adaptive goals, since an emotion is felt any time an adaptively important goal is, or is likely to be, fulfilled or thwarted (Castelfranchi, 2000; Darwin, 1872; Frijda, 1986). Fear monitors the goals of survival and safety, anger the goal of justice, shame the goals of image and self-image.

2. Emotions activate goals: They have a high motivating power in that they trigger goals. Each emotion, along with feelings, physiological arousal, expressive pattern, includes a readiness to action, that is, a goal of high priority that presses to be urgently fulfilled. Fear activates fight or flight, anger triggers aggression, and compassion triggers help.

3. Emotions can become goals: Pleasant emotions generate a goal of feeling them again, while unpleasant emotions a goal of not feeling them next time. If I felt proud because I made my home assignment well, I'll have the goal to study hard again, if I felt ashamed after having aggressed a friend I'll have the goal of not doing so anymore. Thus, emotions result in a learning mechanism.

As we mentioned, persuasion is a way to influence people, that is, to lead them to pursue some goals, and to induce the persuadee to do so the persuader must convince him that the proposed goal is a goal of high value, also because it is a means for other very valuable goals. But to activate a goal in a person not only through rational but also through an emotional mechanism is not the same. Suppose the same course 
is taught by two teachers: one simply teaches me the discipline, but the other also gives me enthusiasm for that discipline; I will probably choose the latter, because attending the former course only fulfils my cognitive goal, while this also fulfils my affective goals. In the same vein, in persuasion attaching the proposed goal only to rational goals of the persuadee is not as effective as attaching it also to emotional goals. Since emotions are linked to biologically adaptive goals, they add a higher value to other goals.

\section{The Persuasive Body}

The importance of body behaviour in persuasive discourse has been stressed ever since ancient rhetoric. Cicero in "De oratore" and Quintilian in "Institutiones Oratoriae" showed how gesture, face, gaze, and posture are an important part of "Actio" (discourse delivery). Especially gestures were studied, due to their capacity of summoning, promising, exhorting, inciting, prohibiting, approving, and to their ease in expressing emotions, showing attitudes, indicating objects of the orator's thought. Quintilian's work about gestures is partly guided by normative intents - he often stresses what gestures should not be used by an orator, while they are - and just because they are - typical of comic actors; but he does so on the basis of a deep and detailed knowledge of the gestures' forms and meanings. For every gesture he also tells us in what segment of the rhetorical structure of discourse it can be used, which means that also gestures, as words, are subject to rules, and their distribution is determined by context. Moreover, from his description one can see that particular combinations of movements with the same hand shape quite precisely convey the meaning of specific speech acts - for example, we "lower [our hands] in apology or supplication (...) or raise them in adoration, or stretch them out in demonstration or invocation" (15) - or express emotions: "we sometimes clench the hand and press it to our heart when we are expressing regret or anger" (104). Finally, Quintilian acknowledges that sometimes through gestures we may induce persuasive effects: "Slapping the thigh (...) is becoming as a mark of indignation, while it also excites the audience" (123).

\subsection{Persuasion and Gesture}

In present times, while a huge quantity of studies address the use of gesture and other bodily signals in everyday conversation, only part of them is devoted to analysing them in persuasive discourse. Some overview some aspects of the body's relevance in political communication (Atkinson, 1984) or focus on the synchronisation of gestures with pauses and intonation and other rhetorical devices, frequently used to quell the applause (Bull, 1986). Others investigate the audience's physiological, cognitive, and emotional reactions to the politicians' facial expression and other vocal and bodily behaviours (Bucy and Bradley, 2004; Frey, 2000). Some recent 
works finally provide detailed morphological and semantic descriptions of gestures and on this basis give pertinent insights about the relation between gesture and persuasive discourse (Tournier, 2003; Kendon, 2004; Streeck 2008).

In her book "The Gestural Expression of a Politician's Thought" (Tournier, 2003), Calbris analyses the political discourses by Lionel Jospin from July 1997 through April 1998 - his first months as a prime minister. Through an insightful analysis of the metaphors exploited by his manual behaviour, she demonstrates how Jospin's gestures or aspects of their execution - for example, the shape of the hand or even which hand is used, right or left - can express abstract notions like effort, objective, decision, balance, priority, private or public stance. At the same time, though, they fulfil discursive functions: they can delimit or stress, enumerate or explicate the topics of discourse.

Kendon (2004) analyses gestures in different cultures and different types of interaction, by people that tell about their past life or comment on everyday life events, sometimes also with an argumentative intent, and distinguishes three main functions of co-verbal gestures: a referential function, of conveying parts of the propositional content of an utterance; an interactive function, of helping the turn-taking management; and a pragmatic function; within this, a gesture has a performative function if it clarifies the type of speech act that is being performed; a modal function if it alters the interpretation of the utterance, e.g. through negation or intensification; and a parsing function, marking the syntactic or textual structure of a sentence or discourse. Then Kendon analyses some gesture families, by singling out, for each specific hand shape and orientation, typical contexts of use and finding unifying semantic themes. Some of the gestures he analyses can well have a persuasive use: for example, the "ring" gestures that bear a meaning of "making precise" or "clarifying" are used every time this clarification is important "in gaining the agreement, the conviction or the understanding of the interlocutor" in Italian culture (p. 241).

\subsection{Persuasion, Body Language, and Politics}

Streeck (2008) analyses the gestural behaviour of the Democratic candidates during the political campaign of 2004 in the USA. They do not use many different gestures, as to hand shape and movement pattern, and the gestures they use are very rarely iconic: partly because, as anticipated by Quintilian, iconics look too much a popular style of gesturing and partly because their function in political discourse is not to convey referential but mainly pragmatic information. Yet, among the candidates' gestures with pragmatic functions, Streeck maintains they mainly fulfil a parsing function, more than a performative one, and they do not unequivocally indicate which speech act is being performed, since they do not imply a fixed form-function relationship. For example, Streeck doubts that the "ring" always has a meaning of precision or that the "power grip" of moving the fist always conveys an assertion of power. In maintaining that the candidates' gestures mainly have a parsing function, 
Streeck (2008) further argues that the tempo of a gesture, more than other aspects of it, is a clear cue to the discourse structure: for example, the alternation of rapid and slow beats, or whether the stroke combines with the peak syllable or with all stressable syllables, distinguishes between background and foreground information.

Poggi and Pelachaud (2008) analysed fragments of three pre-electoral debates through an annotation scheme that described gestures as to their global meaning and to the meaning borne by their expressivity parameters and classified the meanings as to their persuasive import. Then they computed the quantity of persuasive gestures in the two candidates and their percentage of distribution across the various persuasive strategies. The persuasive strategies adopted are somewhat different between the two politicians. They differ as to the proportion of pathos and logos, for both the majority of gestures pursue an ethos strategy and both tend to project more an image of competence than one of benevolence, but for one of the two candidates the preference for the image of competence is much higher than that of benevolence. Moreover, the persuasive strategies are not always conveyed by the gesture shape, but in some cases only by the gesture expressivity or simply by an indirect meaning of the gesture. Pathos is contained more typically (exclusively, for one candidate) in the expressivity of gesture, while ethos in both orators is more often conveyed by the gesture as a whole. Further, the pathos strategy comes out only at the indirect level in both politicians, while they differ in their level of indirectness. From the analysis it resulted that the differences how much each persuasive strategy is exploited and how it is conveyed (through whole gesture, or expressivity, direct or indirect meaning) are congruent with either the context of the specific debate under analysis, or the general political strategy of the candidates, or finally with his general communicative style.

Poggi and Vincze (2008) analysed the gaze behaviour of two orators, Romano Prodi and Ségolène Royal, in political debates and interviews. The study analysed the general persuasive structure of some fragments in terms of hierarchy of goals and the items of gaze through an annotation scheme that analysed their direct and indirect persuasive import. Prodi's gaze was found to use a competence strategy much more frequently than other strategies and more than Royal (67 versus 27\%), while he never used a benevolence strategy. Royal, instead, used the logos gaze most frequently (54\%). Also in this study it was found that the pattern of gaze persuasive strategies of each orator was congruent with the politicians' global political strategy and with the context of the specific debate. This seems to show that during persuasion all body modalities coherently cooperate to one and the same persuasive goal.

\subsection{Quality of Persuasive Body Behaviours}

In a recent study, Poggi and Pelachaud (2008) wondered if it is possible to single out some gesture that one could define as "persuasive gestures". Actually, there could be some - very rare - gestures that one could call persuasive: for example, a gesture of incitation. Yet, it is more frequent that persuasiveness in a gesture does not dwell 
in the global meaning of the gesture per se, but rather in the expressivity of gesture (Hartmann et al., 2002): its spatial extent, temporal extent, fluidity, power, and repetition. Later, Poggi and Vincze (2008) put the same question about the existence of "persuasive gaze" (as described in the previous section).

But what does it mean that a gesture or a gaze is "persuasive"? According to Poggi and Pelachaud (2008), a gesture is persuasive when either the global meaning of the gesture or the meaning of some parts or aspects of it conveys some of the semantic contents that are contained in the persuasive structure of a discourse. Poggi and Pelachaud (2008) and Poggi and Vincze (2008) adopt the model of persuasion of Poggi (2005), according to which persuasion is an act of social influence brought about by communication, through which a persuader $\mathrm{A}$ aims to convince a persuadee B (that is, to make him believe with a high level of certainty) that a goal GA proposed by A is a subgoal to some goal GB that B already has; thus, A must convince $B$ that GA is a goal of high value, possibly of higher value than alternative goals GC or GD, and must do so by exploiting the three Aristotelian strategies of logos (rational argumentation), pathos (induce emotions in B), and ethos (look credible and reliable to B). More specifically, A must show that they are certain of what they are saying (certainty), that they propose goal GA not out of their own concern but in order to achieve the goals of B (benevolence), and that they have good capacities of action and planning to pursue their own goals and to advise goals to others (competence).

Therefore, the gesture and gaze items that have a persuasive import are those that convey the following types of information:

1. Importance. The signals conveying the meaning "important" mention the high value of a proposed goal, thus trying to convince the persuadee to pursue it. This meaning is typically contained in some performative gestures, like incitation and request for attention, or other gestures like Kendon's (2004) "grappolo" ("finger bunch") that convey a notion of importance as their very meaning; but "important" is also the core meaning of beats, since every beat stresses a part of a sentence or discourse, hence communicating "this is the important part of the discourse I want you to pay attention to and to understand". Finally, this can be the meaning of some particular aspects of the expressivity of gesture, for instance irregularity or discontinuity in movement (Chafai et al., 2007).

2. Certainty. To induce certainty in the persuadee, the persuader must show selfconfidence and be certain about what he is saying. This is why gestures that convey high certainty, like the "ring" mentioned by Kendon (2004) and Streeck (2008), may be persuasive. Yet, since persuading can mean either to convince to believe something or to convince to do something, the gestures that convey a high degree of certainty generally persuade to believe and only indirectly persuade to do something.

3. Evaluation. To express a positive evaluation of some object or event implies that it is a useful means to some goal; thus, to bring about that event or to obtain that object becomes desirable, a goal to be pursued. In a marketplace, to convince someone to buy a food, a "cheek screw" (rotating the tip of the index finger on 
cheek), that means "good", "tasty", made by an Italian grocer, would be a good example of persuasive gesture. Of course, we cannot find an example like this in our fragments, due to obvious reason of social register. However, as we see below, a persuader, to pursue an ethos strategy, can make gestures that induce a positive evaluation of him.

4. Sender's benevolence. In persuasion not only the evaluation of the means to achieve goals is important, but also the evaluation of the persuader: the sender's ethos. If I am benevolent to you, you can trust me, so if I tell you that a goal is worthwhile you should pursue it. A gesture driven by the ethos strategy of showing one's moral reliability is, for example, putting one's hand on one's breast, which means "I am noble, I am fair". This gesture is quite frequent in various corpora of political communication (see, for example, Poggi and Pelachaud, 2008; Serenari, 2003).

5. Sender's competence. If I am an expert in the field I am talking about, if I am intelligent, efficient, you might join me and pursue the goals I propose. For example, in a pre-electoral debate a candidate, in talking of quite technical things concerning taxes, rotates his right hand curve open, with palm to left, twice. This gesture means that he is passing over these technicalities, possibly difficult for the audience; but at the same time the relaxed appearance of his movement lets them infer that he is smart because he is talking of such difficult things easily, unconstrained. This provides an image of competence in what he is talking about.

6. Emotion. If I express an emotion, and this is transferred to you through contagion (Poggi, 2004), since emotions trigger goals, a goal will be activated in you, thus implementing a pathos strategy. Another candidate says, "I cannot pretend to act in a country different from what it is", referring to his country, Italy. The movement of his forearm shows low spatial extent and fluidity (it is short and jerky) and high power and velocity, thus conveying an emotion load that aims to transmit a sense of pride of being Italian and thus to elicit the Italians' desire to vote for him. Another candidate in France observes that her opponent's politics towards the unemployed is somewhat punitive. While saying "sanctionner les chômeurs" (punish the unemployed) she raises the internal parts of eyebrows, thus performing a gaze of sadness. Thus she exhibits an emotion and aims to transmit it to the audience: a use of the pathos strategy.

\section{The Effects of Persuasion}

So far we have seen what persuasion is on the part of the persuader; now we can wonder about the effects of body behaviour on persuasion and about how persuasive effects can be assessed during interaction.

As to what are the effects of gestures on persuasion, several authors make the hypothesis that a persuasive discourse is more or less effective depending on the type of gestures used. Typically, as Henley (1977), Burgoon et al. (1990), and Carli et al. (1995) maintain, self-adaptors - the gestures of touching one's body - seem 
to have a negative effect on persuasion. However, presumably it is not so much the type of gesture - whether symbolic, iconic, self-adaptor, object adaptor, or the like that makes the difference in persuasiveness. Our hypothesis is, instead, that it is not the type of gesture that takes persuasiveness away from discourse, but the gesture's meaning. Why, for example, are self-adaptors typically self-defeating in persuasive gesture? Probably because what a self-adaptor lets you infer is that the orator is not very self-confident, that he needs to reassure himself; and this lowers trust in the persuader. One case in which the very meaning of a gesture had a negative effect on persuasion concerns an American candidate, Howard Dean, who used to make a single gesture, "index up", or "finger wag", which seemed to have a subtly selfdefeating effect. According to Streeck (2007), this gesture displayed the speaker's claim that what he was saying was important and instructive; but since Dean was enacting this "hierarchical act" in permanence, he might have given the impression he was presenting himself as one of "superior knowledge", thus spoiling, with a body behaviour somehow contemptuous towards the audience, the ascendancy credited to him by his early textual presence. Also in this case, therefore, what has negative effects on persuasion is not simply the type of gesture used, but its specific meaning.

\section{Assessing the Effect of Persuasion: The Role of Backchannel}

The latter issue to investigate is, How can the persuader monitor if and how much his persuasive effort has been effective? To this goal, the interlocutor's feedback must be taken into account.

When conversing, we try to get our interlocutors to engage with us in a joint project. We utter words, so that they will be heard, listened to, understood and that the other person engages in the proper acts that we are soliciting: attending, understanding, and reacting appropriately; answering our questions; believing our statements; taking up our orders; etc.

Conversational actions are undertaken to engage the addressees in taking actions in turn. When advising people, we hope people take the advice to heart. When cheering them up, we hope they feel happy for a while. When we argue in favour of a certain proposition, we hope that the other will become convinced of what we believe to be true.

In persuasive or argumentative conversations, just like with any action, it is important for speakers to check to what degree these actions are successful on all levels. In the case of conversations these checks involve monitoring the interlocutor to see his or her uptake of the joint projects proposed by the speaker. Through typical behaviours involving gaze, facial expressions, head movements, posture, and vocal backchannels listeners show that they are engaged in the conversation, paying attention, showing they are interested in what is being said, and, in the case of persuasive dialogue, whether they are starting to get convinced or not by the arguments. The interaction between speaker and audience is claimed to be essential in argumentative discourse according to van Eemeren and Grootendorst (1984). 
In ordinary conversational situations some speech acts by speakers are specifically calculated to elicit from listeners certain verbal (and possibly also non-verbal) responses in which they indicate understanding and (in particular) acceptance. In our view this applies pre-eminently to the argumentation advanced during a discussion or debate. This means that to a certain extent arguments in debates are designed to achieve precisely defined verbally externalized illocutionary and perlocutionary effects that are immediately related to the speech acts performed. [p. 24]

A particular type of response to speech is performed through backchannels. These were originally characterised by Yngve (1970) as follows:

[B]oth the person who has the turn and his partner are simultaneously engaged in both speaking and listening. This is because of the existence of what I call the back channel, over which the person who has the turn receives short messages such as "yes" and "uh-huh" without relinquishing the turn. The partner, of course, is not only listening, but speaking occasionally as he sends the short messages in the back channel. The back channel appears to be very important in providing the monitoring of the quality of communication.

As a subset of feedback expressions, these backchannel signals in this view are short vocal messages that have an important structural function (monitoring the quality of the communication). Kendon (1967) talks about a similar category of expressions which he terms accompaniment signals: "short utterances that the listener produces as an accompaniment to a speaker, when the speaker is speaking at length". He divides these into two functional groups: attention signals (in which one appears to signal no more than that one is attending) and assenting signals that express "point granted" or "agreement". In general listener responses can serve several feedback functions. Allwood et al. (1992) distinguish four kinds: contact, perception, understanding, and attitude.

1. Contact: signals that show whether the interlocutor is willing and able to continue the interaction

2. Perception: behaviours that indicate whether the interlocutor is willing and able to perceive the message

3. Understanding: actions that display whether the interlocutor is willing and able to understand the message

4. Attitude: reactions that tell whether the interlocutor is willing and able to react and (adequately) respond to the message, specifically whether he/she accepts or rejects it.

The attitude-feedback functions, of which the assenting signals discussed by Kendon (1967) form an important subset, are interesting for the study of persuasive discourse as they might indicate the success achieved by the person who is trying to persuade.

In real-life examples it is not always easy to say which function is served by a particular feedback expression and many will combine several functions. Within the annotations of the AMI corpus (Jaimes et al., 2007), a difference was made between different types of speech acts that were mainly or exclusively used as listener responses: backchannels that acknowledge reception, 
CommentAboutUnderstanding that indicates understanding or problems with understanding, and assessments. The latter are defined as follows:

An assessment is any comment that expresses an evaluation, however tentative or incomplete, of something that the group is discussing. [...] There are many different kinds of assessment; they include, among other things, accepting an offer, expressing agreement/disagreement or any opinion about some information that's been given, expressing uncertainty as to whether a suggestion is a good idea or not, evaluating actions by members of the group, such as drawings. [...] It can be very short, like "yeah" and "ok".

Assessments are clearly used for the attitudinal reactions, where the speaker expresses his stance towards what is said, either acceptance or rejection. ${ }^{1}$ It thus appears that there is much polysemy ${ }^{2}$ in a verbal backchannel signal that can only in part be resolved by the context. In Sect. 6.1 we take a look at work on non-verbal backchannels that are important in a persuasive context for an ECA.

\section{Towards Persuasive Embodied Conversational Agents}

Embodied conversational agents, ECAs, are virtual human-like entities, with the ability to communicate with users and/or virtual agents. They are endowed with verbal and non-verbal communicative means, such as speech, voice intonation, gesture, body posture, facial expression, gaze. Human users have a tendency to apply the same principles when communicating with other humans or with ECAs (Reeves and Nass, 1996). ECAs are often used as dialogue partners. They can be used as personal assistant, web presenter, companion, tutor, and so on. As pointed out in the previous sections, persuasion may be relevant in each of these applications.

Several models of ECAs have been implemented. While few models have been elaborated for persuasive ECAs, several works have been undertaken to endow ECAs with communicative qualities that allow them to be persuasive in given situations. In the following we present a state of the art of such models. As described above, we have highlighted three qualities that are important in persuasion, namely gesture and expressive gestures, persuasion and emotion, and finally persuasion and backchannel. We now present the existing models in relation to these three qualities.

\footnotetext{
${ }^{1}$ A question looked at by Heylen and op den Akker (2007) was whether it is possible to distinguish an utterance containing "yeah" which expresses a stance (of partial agreement, i.e. an assessment in terms of the AMI annotation scheme) from an utterance that is simply meant as a backchannel. They achieved correct classification only for $60 \%$ of the cases when not taking into account the speech act of the previous utterance and $80 \%$ if they did.

${ }^{2} \mathrm{We}$ avoid the use of the term ambiguity here as the distinction between the various categories is not strict and acknowledgements can easily shift into an assessment.
} 


\subsection{ECA - Persuasion and Gesture}

Not much work has been done so far in implementing gesture features for persuasion in ECA. We report works done in modelling gesture style and expressive gestures that could be used in persuasive strategies as described in Sect. 3 .

Ruttkay and her colleagues (2003) proposed a behaviour representation to encompass styles. An ECA is described over a large set of dimensions ranging from its culture and profession to its emotional and physical state. All these dimensions affect the way an ECA moves and gesticulates. EMOTE (Costa et al., 2000) implements the Laban annotation scheme for dance to change, through a set of parameters, the way a gesture looks depending on values such as the strength of the gesture and its tempo. EMOTE works as a post-filter after a gesture animation has been computed and adds expressivity to its final animation.

A model of behaviour expressivity using a set of six parameters that act as modulation of behaviour animation has been implemented (Hartmann et al., 2005). When applied at these different levels, expressivity may convey different functions in the discourse context: it can attract the attention (Chafai et al., 2007), persuade the addressee (Poggi and Pelachaud, 2008), and indicate emotional state (Martin et al., 2005). In these studies they were interested in understanding and modelling how emotion as well as persuasion can be conveyed qualitatively, in particular through gesture expressivity.

Two-dimensional cartoons were manually annotated to understand how animators used characters' movement expressivity to call for the attention of the spectators. Two types of modulation of behaviour expressivity (irregularities and discontinuities) were found to be used by the animators. They were integrated in an ECA system (Chafai et al., 2007). An evaluation study was conducted to see if these modulations played a role in attracting the user's attention when conversing with the agent. The results of the evaluation confirm that expressivity specified at gesture phase level may play a specific function; namely, here, it can act to attract the attention of the interlocutor at precise moments of the dialogue.

Expressivity parameters were extracted either manually (Devillers et al., 2005; Martin et al., 2005) or automatically (Caridakis et al., 2006) over a whole sequence and played back by an agent. The purpose of these studies was to understand which elements of behaviours, in particular behaviour expressivity, play a role for perceiving an emotional state.

\subsubsection{Persuasion and Backchannel}

In a series of studies Bevacqua et al., (2007) and Heylen et al., (2007), looked at the perception of facial expressions of attitudes that a conversational agent displayed when listening. They looked at a particular subset of expressions that might be relevant to use in persuasive dialogues, where the agent as listener could indicate its understanding or acceptance of the utterances of the speaker.

In a perception test, subjects were asked to watch the virtual character Greta (Bevacqua et al., 2007a) displaying a combination of facial signals and head 
movements. The following behaviours were presented in the 14 video clips shown in a first experiment (Bevacqua et al., 2007b):

1. a single head nod $(\mathrm{N})$

2. a head nod with a smile (NS)

3. a head nod and a raise of the eyebrows (NRE)

4. a head shake (HS)

5. a head shake and a frown (SF)

6. a head shake, a frown, and a tension in the lips that tighten and get thinner (SFT)

7. a frown and a tension in the lips that tighten and get thinner (FT)

8. a raise of the left eyebrows (RLE)

9. the eyes roll in the head (ER)

10. a head tilt to the left with sad eyebrows (TSE)

11. a head tilt to the left and a frown (TF)

12. a head tilt to the right and a raising of the eyebrows (TRE)

13. a head tilt to the right, gaze turns down to the right (TG)

14. eyes wide open (EWO)

The displays are prototypically associated with a number of meanings in a conversational setting as listener responses that are either used as conventional signals or as extensions of the meanings these displays have in, for instance, emotional expressions.

The subjects had to associate one or more meanings to these signals. They could choose from a close list of meanings. In particular, we have investigated how well these expressions matched various classes of performative displays and epistemic and affective states: agreement, disagreement, acceptance, rejection, interest and boredom, believe, disbelieve, understanding, and lack of understanding. These are assumed to be important attitudinal reactions in a persuasive dialogue. One could assume that signals containing nods and smiles would be mostly associated with the positive states whereas shakes, frowns and lip tension would be associated with negative states (Bassili, 1979). Table 1 shows for each behaviour the meaning that was most often assigned to it. In some cases there were two meanings that were equally often chosen.

This study (Heylen et al., 2007) shows that for most attitudes fairly clear prototypical visual expressions can be found. A one-to-many mapping can be established between a facial signal and a backchannel meaning. The results of the study are in line with existing literature. Nods, for example, are mostly used in the contexts of acknowledgements or agreements or as a positive answer substituting or coinciding with the utterance "yes" or an equivalent. Shakes, on the other hand, have a negative meaning (Kendon, 2003). Head tilts often suggest interest. Smiles occur a lot in conversations. They mostly show some positive attitude, except in cases such as a sarcastic grin. Eyebrow movements have a range of meanings. One of the meanings that Ekman (1979) points out is one where it can function as an agreement response indicating that the listener is attending but also understands and does not disagree 
Table 1 Meanings associated with head behaviours

\begin{tabular}{ll}
\hline Nod & Acceptance \\
Nod smile & Liking \\
Nod right eyebrow & Agreement \\
Raise left eyebrow & Disbelieve \\
Tilt sad eyebrows & Not understand \\
Eye roll & Disbelieve \\
Tilt, gaze down & Boredom \\
Shake, frown, tense lips & Disagreement/rejection \\
Frown, tense lips & Disagreement \\
Head shake & Rejection \\
Shake, frown & Disagreement, rejection \\
Tilt, frown & Disagreement, disbelieve \\
Tilt right, raise brows & Believe (is this correct) \\
Eyes wide open & Disbelieve \\
\hline
\end{tabular}

with what is being said. Raising of the eyebrows together with a head nod or an agreement word is a typical agreement listener response. However, raised eyebrows can also figure in displays of surprise. Also a frown can function as an expression of perplexity but is also associated with expressions of anger and disgust. The perplexity that is often being expressed by the frown can function as a response to the speaker indicating lack of understanding or indicating that one thinks what is being said does not make sense.

\subsection{Persuasion and Emotion}

In persuasion one has to convince with logos (rational argumentation), ethos (the persuader's credibility and reliability), and pathos (the appeal to the emotions of the interlocutor). In both trying to be convincing and transmitting emotions can one need to exhibit a fake personality and/or fake emotions? In human-human communication, people often try to hide their real emotions because the social situation requires it. Typical example are excuses, such as "I would love to join you, but ...". Emotions are the number-one topic that people lie about and studies show that up to $30 \%$ of social interaction longer than 10 min contains such deceptions (DePaulo et al., 1996). Endowing technical systems like embodied conversational agents with the ability to detect, represent, generate, and/or show emotions, it is thus indispensable to investigate the crucial questions how to handle false emotional expressions from the user and how and when to create false emotional expressions in the ECA.

\subsubsection{Persuasion and Deception}

Various attempts have been made to create synthetic agents that deliberately oppress or express a certain emotion. De Rosis and colleagues (2003) as well as Prendinger and colleagues (2001) have developed agents that are able to control their emotional 
displays if the social situation requires it. For instance, if the social distance between an agent and its conversational partner is high, Prendinger's agent would not show anger to the full extent. The virtual tutor COSMO developed by Lester and colleagues (2000) intentionally portrays emotions with the goal of motivating students and thus increasing the learning effect.

Earlier approaches start from the assumption that the agent is able to perfectly hide emotions if the social or pedagogical situation requires it. However, humans are not always capable of completely concealing their true emotions. For instance, masking smiles cannot entirely override the muscular programme of the original emotion because not every facial muscle can be consciously controlled. As a consequence, such a mask will always include segments of one's felt emotion. The question arises as to how to handle situations in which the agent decides to display an emotion which is in conflict with its internal appraisal processes. In some situations, it might be desirable to employ agents that perfectly convey "wrong" emotions with the aim to convince the interlocutor. Consider a sales agent on the web that has to advertise a product of minor quality. If it does not succeed in concealing its negative attitude towards the product, a decrease of the sales might be the consequence. On the other hand, agents in social settings may come across as little believable or cold if they are always able to perfectly hide their true emotions. In addition, the display of mixed emotions may even lead to a positive response from the interlocutor. For instance, students may feel sympathy towards a virtual teacher that desperately tries to hide its negative emotions caused by the students' bad performance. Last but not least, the emulation of deceptive behaviours may enrich our interactions with synthetic agents - especially in game-like environments.

Following these considerations, Rehm and André (2005a) focused on synthetic agents that may express emotions that are in conflict with their appraisal processes. Unlike earlier work, they modelled situations in which the agent fails to entirely conceal its "felt" emotions. They developed an agent whose behaviours may reflect potential conflicts between "felt" and deliberately expressed emotions. Their work concentrated on facial expressions of deception which have been profoundly researched in the psychological literature. According to Ekman and colleagues (1988), there are at least four ways in which facial expressions may vary if they accompany lies and deceptions: micro-expressions, masks, timing, and asymmetry.

1. Micro-expressions: A false emotion is displayed, but the felt emotion is unconsciously expressed for a fraction of a second. The detection of such micro-expressions is possible for a trained observer.

2. Masks: The felt emotion (e.g. disgust) is masked by a noncorresponding facial expression, in general by a smile. Because we are not able to control all of our facial muscles, such a masking smile is in some way deficient. Thus, it reveals at least in part the original emotion.

3. Timing: Facial expressions accompanying true emotions do not last for a very long time. Thus, the longer an expression lasts the more likely it is that it is accompanying a lie. A special case seems to be surprise, where elongated onand offset times are a good indicator of a false emotion. 
4. Asymmetry: Voluntarily created facial expressions like they occur during lying and deceiving tend to be displayed in an asymmetrical way, i.e. there is more activity on one side of the face than on the other.

To model the non-verbal behaviour, they employed the Greta agent system developed by Pelachaud and colleagues (2007). For example, Fig. 1 shows the facial displays for genuine joy, genuine disgust, and disgust faked by joy. In the case of natural joy (left screenshot), the corners of the mouth and cheeks are moved up symmetrically. In the case of natural disgust (middle screenshot), the eyebrows are contracted and the upper lip is moved up. To fake disgust by joy (right screenshot), the eye movements of disgust and the mouth movements of joy have been combined. Different degrees of masking are combined with different degrees of asymmetry of the facial displays resulting in 32 possible facial expressions.

A computational model of complex facial expression was developed by Niewiadomski and Pelachaud (2007a, b). It is also based on Ekman's work (1975, 2003). Complex facial expressions are obtained using a face partitioning approach where facial expression is defined by a set of eight facial areas: forehead/eyebrow, upper eyelid, eyes, lower eyelid, cheek, nose, lip (movement), and lip tension. In particular, in a case of complex facial expressions different emotions can be expressed on different areas of the face. The complex facial expressions are composed of the facial areas of input expressions using a set of fuzzy rules. Different rules have been implemented for the superposition of two felt emotions (see Fig. 2) and for the masking of a felt emotion by a fake (i.e. non-felt) emotion (see Fig. 3). The rules make use of the results showing that expressions of felt emotions are signalled by characteristic (also called reliable) features (such as the crow feet for felt happiness) $(1975,2003)$ and that positive emotions are mainly perceived from the lower part of the face (smile for happiness) and negative emotions from the upper face (such as frown for anger) (Bassili, 1979).

An example of such a rule for the superposition of two felt emotions (sadness and anger) is the following the more one of the input expressions is (similar to) anger and the other input expression is (similar to) sadness, the more certain is that the final expression contains brows, upper eyelids, eyes, and the lower eyelids of the first expression and the mouth area rest of the second (see Fig. 2). The output of this module is a facial expression composed of parts of the facial expression of one emotion and parts of the facial expression of the other emotion. They refer to this final expression as a complex expression as it is composed of two expressions and thus shows two emotions.

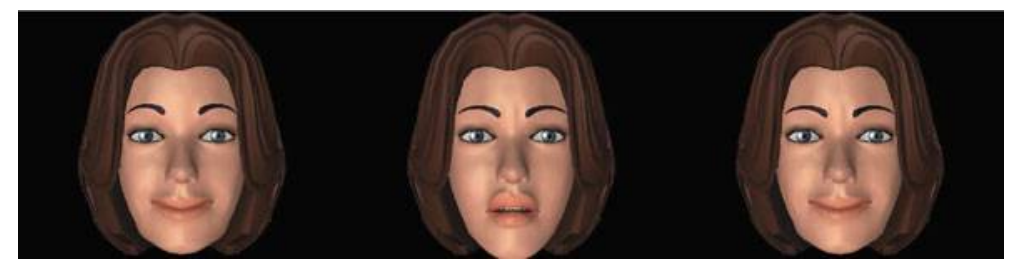

Fig. 1 Greta showing genuine joy (left), genuine disgust (middle), and disgust faked by joy (right) 

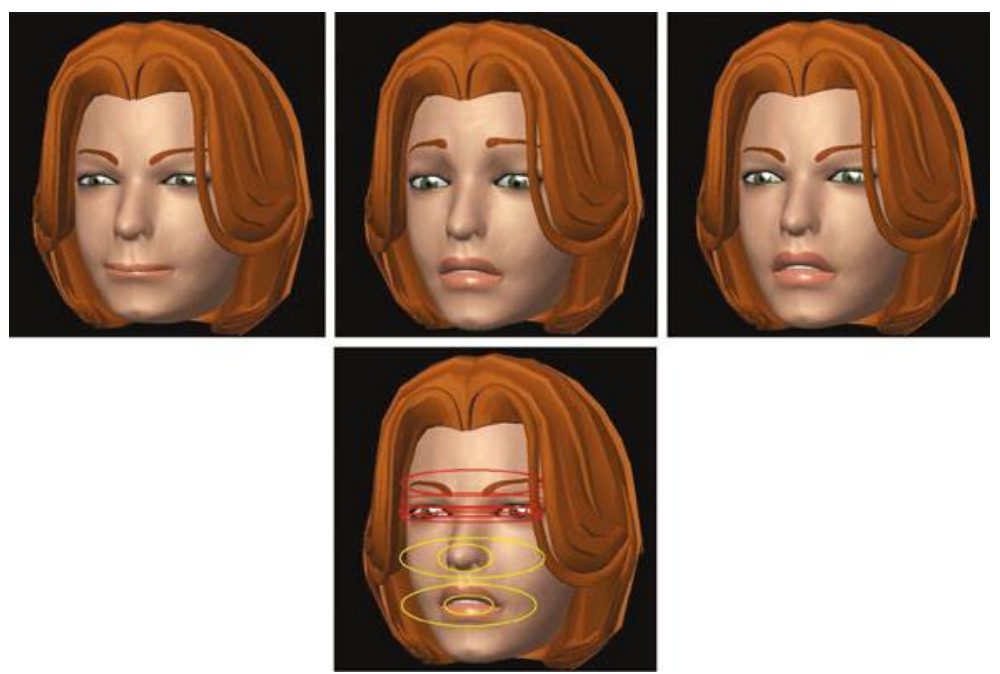

Fig. 2 Superposition of anger and sadness. From the left to right: anger (a), sadness (b), superposition of anger and sadness (c) superposition of anger and sadness with significant areas marked (d)
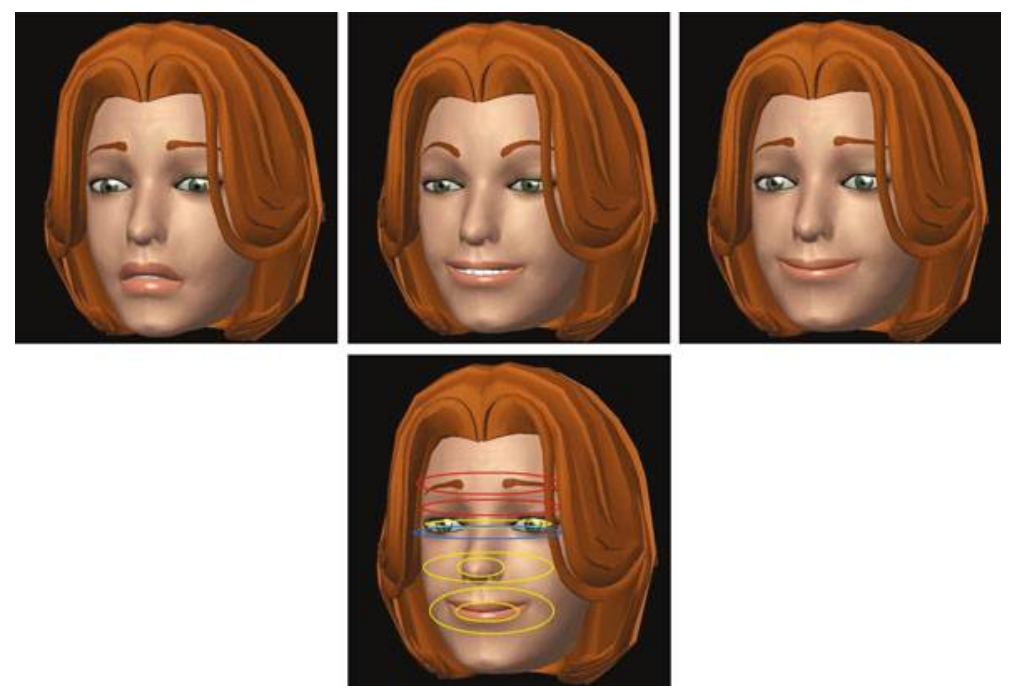

Fig. 3 Sadness masked by happiness. From the left to right: sadness (a), happiness (b), sadness masked by happiness (c) sadness masked by happiness with significant areas marked (d)

Figure 3 presents the agent displaying an expression of sadness, that is, masked by a fake happiness. According to these rules facial areas of forehead/eyebrows and upper eyelids cover the features of felt sadness (red circles in Fig. 3d) that leak over the mask of a joy (yellow circles in Fig. 3d). As a consequence, they can be observed in a final expression. 
To find out how deceptive clues are subjectively perceived by a human user and to what extent users are able to correctly interpret them, Rehm and André (2005a) conducted two experiments. In the first experiment, a deceptive and a non-deceptive version of the Greta agent presented movie reviews to the users. That is, user did not interact with the agent and information was provided by the agent in terms of a monologue. The study revealed that the non-deceiving agent is perceived as being more reliable, trustable, convincing, credible, and more certain about what it said. Although people reacted to facial clues of deceit when they had the opportunity to carefully watch and compare different instances of agents, they were not able to name the reasons for these reactions.

In the second experiment, the agent was serving as a game partner in a game of dice. To win the game, players had to lie to the other players and to catch liars. In the game, the agent tried to mislead the other players by portraying facial expressions that did not correspond to her actual emotional state. For instance, she expressed false joy to make her game partners believe that she achieved a high score. Nevertheless, the agent did not lie in a perfect manner, but still revealed her deceptive behaviours by subtle facial cues. The first study indicated that even subtle expressions of deception may have an unfavourable impact on the user's perception of the agent. The results of the first study could, however, not be confirmed for the second scenario in which the experimental conditions were much less controlled. Most likely the players were too much engaged in the game to pay attention to the agent's deceptive cues. In a more natural and engaging face-to-face situation, subjects tend to disregard deceptive clues.

\subsubsection{Persuasion and Politeness}

If you want to persuade someone through inducing positive emotions in him/her a good way to do so may be politeness. When humans interact with each other, they risk continuously threatening the face of their conversational partners, for example, by showing disapproval or by putting the other person under pressure. To mitigate such face threats, humans usually rely on politeness tactics. For instance, instead of formulating a direct request "Solve the equation", a teacher might suggest "Why don't we solve the equation together?"

In their seminal work, Brown and Levinson (1987) analyse verbal strategies of politeness. Positive politeness seeks approval of the addressee, for example, by making him a compliment before a criticism is delivered. Negative politeness emphasises the hearer's freedom of action, for example, by formulating a suggestion instead of a request. Off-record statements are vague and the addressee has to infer the exact meaning of the speaker leaving him in the position to misunderstand the speaker and thus to not feel offended. Previous work has concentrated for the most part on verbal means to mitigate face threats. An exception is an empirical study by Trees and Manusov (1998) who found that non-verbal behaviours, such as pleasant facial expressions and more direct body orientation, may help to mitigate face threats evoked by criticism. Bavelas et al., (1995) provide a classification of gestures, some of which can be directly mapped onto Brown and Levinson's strategies of politeness. Shared information gestures mark material that 
is part of the interlocutor's common ground. Citing gestures refer to previous contributions of the addressee and aim at conveying the impression that the interlocutors share a common opinion. Elliptical gestures mark incomplete information that the addressee should augment for himself or herself and may take on a similar function as off-record strategies. Seeking agreement gestures directly correspond to Brown and Levinson's approval-oriented strategies. Turn open gestures can be regarded as attempts to satisfy the addressee's desire for autonomy. Linguistic means to deliver face-threatening acts have partly become part of the grammar and Bavelas classification of gestures suggests that there might be similar principled and standardised connection between non-verbal means of communication and politeness strategies.

To shed light on the question of how face threats are mitigated by nonverbal means, Rehm and André (2005b) conducted a corpus study with human speakers. This multimodal corpus consists of staged interactions of inherently face-threatening situations. They devised a scenario that forced the participants to use their (unconscious) knowledge of politeness strategies by confronting them with an inherently face-threatening situation. To code politeness strategies, they followed Walker et al.'s (1997) categorisation of direct, approval-oriented, autonomy-oriented, and off-record strategies.

The results of their corpus analysis indicate that gestures are indeed used to strengthen the effect of verbal acts of politeness (Rehm and André, 2005b). In particular, vagueness as a means of politeness is reflected not only by verbal utterances but also by gestures. Iconic and deictic gestures were overwhelmingly used in more direct criticism while there was a high frequency of metaphoric gestures in offrecord strategies. Obviously, the subjects did not attempt at compensating for the vagueness of their speech by using more concrete gestures.

Walker et al. (1997) have presented one of the first approaches to implement politeness strategies based on the theory by Brown and Levinson (1987) as a means to more flexible dialogue control. They describe a selection mechanism that is based on the variables power, social distance, and ranking of speech act. Johnson et al. (2004) investigated the potential benefits of politeness in a tutoring system. Examining the interactions between a real tutor and his students, they came up with a set of templates each of which is annotated according to the amount of redress that tactic gives to the learner's face. Rehm and André (2005b) equipped the Greta agent with politeness behaviours based on the corpus study presented above.

Niewiadomski and Pelachaud (2007b) analysed the same video-corpus of Rehm and André (2005b) in order to find relations between politeness strategies and facial behaviour. They considered four types of facial displays: expression of the true emotional state, inhibited, masked, and fake expression. They analysed the frequency of occurrence for each of them and found that different types of facial expressions were not evenly distributed along the different strategies of politeness. They used this information to build their model of facial deceptive behaviour management in interpersonal relations. In their model, they considered three variables to encompass the characteristics of the interaction (Brown and Levinson, 1987) and the emotional state of the displayer, namely social distance, social power, and emotion valence. Based on the value of these variables they defined which facial management is the most appropriate, e.g. when a felt emotion should be masked or inhibited. 


\section{Recommendations to Build a Persuasive ECA}

To conclude this chapter, we provide recommendations for non-verbal behaviours to be considered when constructing a persuasive ECA.

\subsection{Gaze Behaviour}

Eye contact is a powerful tool for persuasive purposes. It can be used to acknowledge presence, show interest, provide feelings of importance, and help convey an attitude that the persuader likes the persuadee, something also influenced by large pupil size. In courtroom situations, making eye contact with jurors has been referred to as being the most important type of communication possible (Bernstein et al., 1994). Speakers who engage in eye contact are perceived as being more credible (Hemsley and Doob, 1978) than those who continually avert their gaze, the latter appearing untrustworthy or unconfident. However, it is important to establish a balance between appearing confident by engaging in sufficient eye contact, while at the same time avoiding to be perceived as overpowering or threatening by maintaining eye contact for too long. This is a difficult proposition in practice, as it must account for many factors related to the context of the interaction, the persuader and the persuadee, such as culture and gender. At a higher level, gaze strategies such as logos gaze, competence, and benevolence strategies (see Sect. 2) can be adopted to support persuasion through rational arguments, establishing trust, and invoking emotions (Poggi and Vincze, 2008). In these situations, a key factor should be to ensure congruence between gaze and other modalities in order to provide coherent persuasive behaviour consistent with a single strategy. The behavioural realism of the gaze behaviour is also a factor for consideration, as virtual humans higher in behavioural realism have been found to be more influential (Guadagno et al., 2007).

\subsection{Facial Expressions}

Facial expressions convey a large amount of non-verbal information: a smile or a frown made at a certain moment can speak volumes. In general, facial expressions should appear to be relaxed in order to help convey a confident but non-threatening demeanour: tension should not be perceptible in the brows, jaw, mouth, or shoulders so that the persuader appears to be in control of the situation at all times.

\subsection{Showing Attention and Interest}

Related to eye contact and facial expression is the importance of showing interest in what the other has to say. For example, as described in Levine (2006), based on his experiences working on the documentary Thin Blue Line, interviewer Errol Morris 
highlighted the importance of appearing to be engrossed and show interest in what his guests had to say at all costs. In some situations, Morris even suggested not actually becoming too engrossed in the story, so one could concentrate totally on providing signals of interest, which he regarded as being of the utmost importance for eliciting disclosure of facts from interviewees.

\subsection{Hands and Arms}

Great speakers use hand gestures more than on average and gestures can give the listener confidence in the speaker as well as help the speaker maintain their turn. It is particularly important that hand gestures keep out of the personal space of the other and especially away from their face. The arms should reinforce the impression of openness on the part of the agent by being uncrossed. As with facial expressions, arm movements should not appear to be tense and should be open and expansive. Humans have a natural tendency to protect vulnerable organs especially in threatening or stressful situations - not doing so can make us feel exposed and vulnerable, but can project a courageous posture, reflecting self-confidence. Furthermore, hands should ideally be visible rather than placed in pockets: Open palms are a good way to express honesty and trustworthiness. Self-touching behaviours are to be avoided, mainly because they may convey a need for self-reassurance.

\subsection{General Posture and Barriers}

As with the other factors, it is important to project an open, honest, cooperative posture, as this can be perceived to reflect a psychological openness. In contrast, a poor, deflated posture is associated with a lack of confidence or a lack of interest. The orientation of the body is of importance here too: as the term "cold shoulder" suggests, when we feel uncomfortable with a person of situation, we may tend to orientate our body sideways, showing aversion of timidity. Orienting towards the audience, and not allowing obstructions between them and the persuader, is of importance in establishing a direct connection with them. Arms, obstacles, and instruments may be used to create barriers, severing this connection and portraying a defensive posture thus, arms should not be placed at rest in front of the abdomen or chest.

\section{References}

Allwood J, Nivre J, Ahlsén E (1992) On the semantics and pragmatics of linguistic feedback. J Semantics 9(1):1-26

Atkinson M (1984) Our masters' voices. The language and body language of politics. Methuen, London

Bassili JN (1979) Emotion recognition: the role of facial movement and the relative importance of upper and lower areas of the face. J Pers Soc Psychol 37(11):2049-2058 
Bavelas JB, Chovil N, Coates L, Roe L (1995) Gestures specialized for dialogue. Pers Soc Psychol Bull 21(4):394-405

Bernstein LE, Demorest ME, Eberhardt SP (1994) A computational approach to analyzing sentential speech perception: phoneme-to-phoneme stimulus-response alignment. J Acoust Soc Am 95(6):3617-3622

Bevacqua E, Heylen D, Pelachaud C, Tellier M (2007a, April) Facial feedback signals for ecas. In: Proceedings of AISB'07: artificial and ambient intelligence, Newcastle University, Newcastle upon Tyne, UK

Bevacqua E, Mancini M, Niewiadomski R, Pelachaud C (2007b) An expressive eca showing complex emotions. In: Proceedings of the AISB annual convention, Newcastle, UK, pp 208-216

Brown B, Levinson SC (1987) Politeness: some universals on language usage. Cambridge University Press, Cambridge

Bucy EP, Bradley SD (2004) Presidential expressions and viewer emotion: Counterempathic responses to televised leader displays. Soc Sci Inf 43:59-94

Bull PE (1986) The use of hand gesture in political speechers: some case studies. J Lang Soc Psychol 5:102-118

Burgoon JK, Birk T, Pfau M (1990) Nonverbal behaviors, persuasion, and credibility. Hum Commun Res 17:140-169

Caridakis G, Raouzaiou A, Karpouzis K, Kollias S (2006, May) Synthesizing gesture expressivity based on real sequences. In: Workshop on multimodal corpora: from multimodal behaviour theories to usable models, LREC 2006 Conference, Genoa, Italy, 24-26 May 2006

Carli LL, LaFleur SL, Loeber CC (1995) Nonverbal behavior, gender, and influence. J Pers Soc Psychol 68:1030-1041

Castelfranchi C (2000) Affective appraisal versus cognitive evaluation in social emotions and interactions. In: Paiva AM (ed) Affective interactions: towards a new generation of computer interfaces. Springer, Heidelberg, pp 76-106

Chafai NE, Pelachaud C, Pelé D (2007) A case study of gesture expressivity breaks. Int J Lang Resour Eval, special issue on Multimodal Corpora Modell Hum Multimodal Behav 41(3-4):341-365

Costa M, Zhao L, Badler NI (2000) The EMOTE model for effort and shape. In: Proceedings of the 27th annual Conference on Computer Graphics and Interactive Techniques SIGGRAPH '00, New Orleans, ACM Press, New York, NY, pp 173-182

Darwin C (1872) The expression of the emotions in man and animals. John Murray, London

DePaulo BM, Kashy DA, Kirkendol SE, Wyer MM, Epstein JA (1996) Lying in everyday live. J Pers Soc Psychol 70(5):979-995

deRosis F, Pelachaud C, Poggi I, Carofiglio V, De Carolis B (2003) From Greta's mind to her face: modelling the dynamics of affective states in a conversational embodied agent. Int J Hum Comput Stud 59(1-2):81-118

Devillers L, Abrilian S, Martin J-C (2005) Representing real life emotions in audiovisual data with non basic emotional patterns and context features. In: First international conference on affective computing and intelligent interaction (ACII'2005), Beijing, China, pp 519-526

Ekman P (1975) Unmasking the face. A guide to recognizing emotions from facial clues. PrenticeHall, Inc., Englewood Cliffs, NJ

Ekman P (1979) About brows: emotional and conversational signals, pages 169-202. Cambridge University Press/Editions de la Maison des Sciences de l'Homme

Ekman P (2003) The face revealed. Weidenfeld \& Nicolson, London

Ekman P, Friesen W, O’Sullivan M (1988) Smiles when lying. J Personal Soc Psychol 54(3): $414-420$

Frey S (2000) Die Macht des Bildes. Der Einfluss der nonverbalen Kommunikation auf Kultur und Politik. Huber, Bern

Frijda N (1986) The emotions. Cambridge University Press 
Guadagno RE, Blascovich J, Bailenson JN, McCall C (2007) Virtual humans and persuasion: the effects of agency and behavioral realism. Media Psychol 10:1-22

Hartmann B, Mancini M, Pelachaud C (2002) Formational parameters and adaptive prototype instantiation for MPEG-4 compliant gesture synthesis. In: Computer animation'02, Geneva, Switzerland, 2002. IEEE Computer Society Press

Hartmann B, Mancini M, Pelachaud C (2005) Implementing expressive gesture synthesis for embodied conversational agents. In: Gesture in human-computer interaction and simulation, 6th international gesture workshop, GW 2005, Berder Island, pp 188-199

Hemsley GD, Doob AN (1978) The effect of looking behaviour on perceptions of a communicator's credibility. J Appl Soc Psychol 8:136-144

Henley NM (1977) Body politics: power, sex, and nonverbal behavior. Prentice-Hall, Englewood Cliffs, NJ

Heylen D, Bevacqua E, Tellier M, Pelachaud C (2007) Searching for prototypical facial feedback signals. In: Pelachaud C, Martin J-C, André E, Chollet G, Karpouzis K, Pelé D (eds) Proceedings of the 7th international conference on intelligent virtual agents (IVA), vol 4722 of Lecture Notes in Computer Science, pp 147-153. Springer, Heidelberg

Heylen D, op den Akker R (2007) Computing backchannel distributions in multi-party conversations. In: Cassell J, Heylen D (eds) Proceedings of the ACL workshop on embodied language processing, vol W07-19, Prague, Czech Republic, 2007, pp 17-24. Association of Computational Linguistics

Poggi I (2004) Emotions from mind to mind. In: Proceedings of the workshop W13: empathic agents. third international conference on autonomous agent systems, AAMAS 2004, New York, NY, USA, pp 208-216

Jaimes A, Bourlard H, Renals S, Carletta J (2007) Recording, indexing, summarizing, and accessing meeting videos: an overview of the ami project. In: Fourth international conference on image analysis and processing (ICIAP 2007) and workshop on visual and multimedia digital libraries (VMDL 2007), Modena, pp 59-64

Johnson WL, Rizzo P, Bosma W, Kole S, Ghijsen M, van Welbergen H (2004) Generating socially appropriate tutorial dialog. In: ISCA workshop on affective dialogue systems, Modena, pp 254-264

Kendon A (1967) Some functions of gaze direction in social interaction. Acta Psychol 26:22-63

Kendon A (2003) Some uses of head shake. Gesture 2:147-182

Kendon A (2004) Gesture : visible action as utterance. Cambridge University Press Cambridge

Lester JC, Towns SG, Callaway CB, Voerman JL, FitzGerald PJ (2000) Deictic and emotive communication in animated pedagogical agents. In: Prevost S, Cassell J, Sullivan J, Churchill E (eds) Embodied conversational characters. MITpress, Cambridge, MA, pp 123-154

Levine R (2006) The power of persuasion. chapter Whom do we trust? Experts, honesty and likability. Wiley

Martin J, Abrilian S, Devillers L (2005) Annotating multimodal behaviors occurring during non basic emotions. In: Proceedings of the first international conference on affective computing and intelligent interaction (ACII), Pkin, Chine

Martin J-C, Abrilian S, Devillers L (2005) Annotating multimodal behaviors occurring during non basic emotions. In: 1st international conference on affective computing \& intelligent interaction (ACII'2005), Beijing, China, 2005 October 22-24

Maurice Tournier (2003) Geneviève Calbris. L'Expression gestuelle de la pense d'un homme politique. CNRS Communication

Mehrabian A (1971) Silent messages. Wadsworth Publishing Company

Niewiadomski R, Pelachaud C (2007a) Fuzzy similarity of facial expressions of embodied agents. In: Pelachaud C, Martin J-C, André E, Chollet G, Karpouzis K, Pelé D (eds) Proceedings of the 7th international conference on intelligent virtual agents (IVA). Lecture Notes in Computer Science, vol 4722. Springer, Heidelberg, pp 86-98

Niewiadomski R, Pelachaud C (2007b) Model of facial expressions management for an embodied conversational agent. In: Paiva A, Prada R, Picard RW (eds) Second international conference 
on affective computing and intelligent interaction (ACII). Lecture Notes in Computer Science, vol 4738. Springer, Heidelberg, pp 12-23

Pease B, Pease A (2006) The definitive book of body language. Bantam Books

Poggi I (2005) The goals of persuasion. Pragmatics Cogn 13:298-335

Poggi I, Pelachaud C (2008) Persuasion and the expressivity of gestures in humans and machines. In: Wachsmuth I, Lenzen M, Knoblich G (eds) Embodied communication in humans and machines Oxford University Press, Oxford, pp 391-424

Poggi I, Vincze L (2008) Persuasive gaze in political discourse. In: Proceedings of the AISB annual convention, Aberdeen, Scotland, UK

Prendinger H, Ishizuka M (2001) Social role awareness in animated agents. In: Proceedings of the fifth international conference on autonomous agents, Montreal, Quebec, Canada, pp 270-277

Quintilianus MF (1920) Institutio Oratoria (trans: Butler HE). Loeb Classical Library. Harvard University Press, Cambridge

Reeves B, Nass C (1996) The media equation: how people treat computers, television, and new media like real people and places. Cambridge University Press, Cambridge

Rehm M, André E (2005a) Catch me if you can - exploring lying agents in social settings. In: Dignum F, Dignum V, Koenig S, Kraus S, Singh MP, Wooldridge M (eds) the proceedings of international joint conference on autonomous agents and multi-agent systems (AAMAS), Utrecht, The Netherlands, 2005, pp 937-944. ACM

Rehm M, André E (2005b) More than just a friendly phrase: multimodal aspects of polite behavior in agents. In: Nishida T (ed) Conversational informatics: an engineering approach. Wiley, Chichester, pp 69-84

Ruttkay Z, van Moppes V, Noot H (2003) The jovial, the reserved and the robot. In: Proceedings of the AAMAS03 Ws on embodied conversational characters as individuals, Melbourne, Australia

Seiter JS, Kinzer HJ, Weger H Jr (2006, April) Background behavior in live debates: the effects of the implicit ad hominem fallacy. Commun Rep 19(1):57

Serenari M (2003) Examples from the Berlin dictionary of everyday gestures. In: Rector M, Poggi I, Trigo N (eds) Gestures. Meaning and use. Edicoes Universidade Fernando Pessoa

Streeck J (2008) A case study of the democratic presidential candidates during the 2004 primary campaign. Res Lang Soc Interact 41:154-186

Trees AR, Manusov V (1998) Managing face concerns in criticism. Hum Commun Res 24(4): $564-583$

van Eemeren FH, Grootendorst R (1984) Speech acts in argumentative discussions. Walter de Gruyter and Foris, Berlin

Walker MA, Cahn JE, Whittaker SJ (1997) Improvising linguistic style: Social and affective bases for agent personality. In: First international conference on autonomous agents, Marina del Rey, CA, USA, pp 96-105

Yngve VH (1970) On getting a word in edgewise. In: Papers from the sixth regional meeting of the Chicago Linguistic Society. Chicago Linguistic Society, Chicago; pp 567-577

Watson K, Mattews B, Allman J (2007) Brain activation during sight gags and language-dependent humor. Cerebral Cortex 17(2)

Yuill N (1997) A funny thing happened on the way on the classroom: jokes, riddles and metalinguistic awareness in understanding and improving poor comprehension in children. In: Disabilities: processes and intervention. Erlbaum, Mahwah, NJ 